\title{
Evaluation Of The Fatigue Strength Of Multiphase Pump Components Under Corrosive Conditions Using A Short Crack Fracture Mechanics Approach
}

\author{
Jochen Hoehbusch $^{1 *}$, Sebastian Bucher ${ }^{1}$, Dr. Henning Haensel ${ }^{1}$, \\ Prof. Dr. Jan Scholten ${ }^{1}$ \\ ${ }^{1}$ Research Group for Offroad Machines, Ruhr-University Bochum, Germany \\ *hoehbusch@Imf.rub.de
}

Keywords: corrosion fatigue, high-cycle fatigue, pitting corrosion, short crack, fatigue limit

\begin{abstract}
Components of Multiphase pumps, employed in oil and gas fields, often suffer from wear and localized corrosion, which is caused by the aggressive media to be pumped. To ensure a safe operation, analytic stress assessments are required. However common guidelines do not include a general way to take the influence of corrosion into account up to now.

Hence this paper presents both a calculation and an experimental method to evaluate the actual fatigue limit under the influence of pitting corrosion for components of stainless steel. The experimental method delivers material-parameters and is used to validate the calculation method. Both of these methods are applied to a weak-notched specimen.
\end{abstract}

\section{Introduction}

Components used in oil and gas production are often exposed to dynamic loads in a corrosive environment. A crack initiation caused by high load frequencies has to be avoided, since component failure can arise in short-terms. These components are usually made of stainless steel in order to prevent corrosive attack. Nevertheless aggressive media can lead to localized corrosion in many cases, i.e. pitting corrosion [2]. Thus an analytic strength assessment is required in order to ensure a safe operation. Up to now, guidelines for analytical strength assessment used in industrial environment, do not include a general way to take the influence of corrosion into account. This leads to the need for a different approach to consider the influence of corrosion on the components fatigue strength.

A special type of corrosion, called pitting corrosion fatigue (PCF), the interaction of pitting corrosion and fatigue is regarded in this paper. PCF starts with the initiation and growth of corrosion pits. Both, initiation and growth of corrosion pits can occur without mechanical stress, so there is no stress value that can be defined as PCF threshold stress $[2,6]$. The growth of the pits leads to a change of the stress state which is characterized by increasing mechanical peak stress and decreasing stress gradients. If the stress reaches a threshold value, cracks are initiated at the corrosion pit. The first initiation of cracks at a corrosion pit is known as pit-to-crack transition [6].

This paper presents an approach to determine the conditions (stress and pit size) for a pit-tocrack transition in components. The pit-to-crack transition is regarded as the end of the safe live of a component which is exposed to high frequency loads. Due to this it may lead to an approximate estimation of the service life. The approach consists of a calculation method with special experimentally derived material parameters. These parameters are determined by an iterative newly developed experimental method using a combination of the shown calculation method and known specimen tests.

\section{Calculation Method}

The basic assumption of the calculation method is the occurrence of the pit-to-crack transition, if the applied stress amplitude at the corrosion pit is greater than the fatigue limit in 
the area of the corrosion pit. The fatigue limit depends on the shape and size of the corrosion pit. Due to the permanent progress of the corrosion pit's growth, the fatigue limit of a particular corrosion state is called actual fatigue limit $\sigma_{D, a c t}$.

For many materials, especially most high-alloy steels, a fatigue limit does not exist [3]. Nevertheless, this value is used in this paper. It is used based on the assumption that the reduction of the fatigue strength is controlled by the corrosion supposing that the corrosion appears much faster than the deterioration due to ultra high cycle fatigue.

Due to the small sizes of corrosion pits, it is impossible to treat them like notches according to e.g. the FKM guideline [4]. Kawai and Karsai [5] regarded them as short cracks, because short crack fracture mechanics do not just cover small cracks, but also small volumetric flaws. They used El Haddads [7] short crack fracture mechanics approach for calculation and compared it with rotational bending fatigue tests. The fatigue tests were carried out under corrosive conditions. The size of the corrosion pit leading to the crack was determined after the final fracture of the specimens.

El Haddads approach does not perform, if the cracks originate in notches [3], therefore, a different approach is used. Fujimoto et al. [8] assume that there is an inherent damage zone of size $a_{\mathrm{s}}$ under the surface. The size of this zone depends on material and range from $0.03 \mathrm{~mm}$ to $0.1 \mathrm{~mm}$ for common steels. Based on this assumption, the stress in a distance $a_{s}$ to the surface of the part and the crack is regarded for the threshold of short crack growth. This stress is the so called effective stress. In this paper Fujimoto's approach is used to calculate the actual fatigue limit. Fig. 1 displays exemplary curves, which show the reduction of the actual fatigue limit depending on the pit size and the stress distance $a_{s}$.

There are three advantages of Fujimoto's

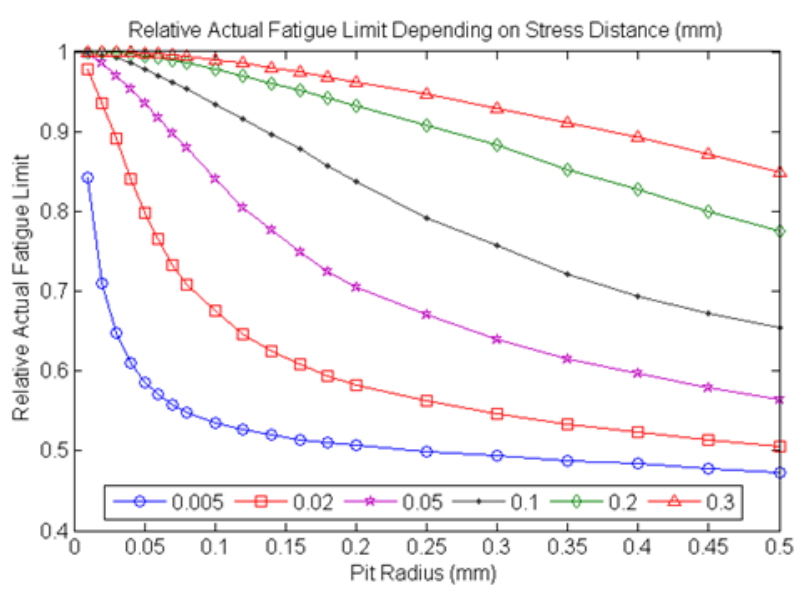

Fig.1: Influence of the Stress Distance on the Actual Fatigue Limit approach. At first is the applicability to notches [3]. Secondly, it allows using common material parameters like the fatigue limit $\sigma_{D}$ and last, the corrosion pits do not have to be substituted with cracks but can be modeled with their original shape.

\section{Application of the Calculation Method}

The proposed calculation method is adapted to weak notched specimens which are made from the stainless steel 1.4462 (yield strength $450 \mathrm{~N} / \mathrm{mm}^{2}$, tensile strength $650 \mathrm{~N} / \mathrm{mm}^{2}-770$ $\mathrm{N} / \mathrm{mm}^{2}$ ). The specimens include a corrosion pit which is modeled as a cylinder with a ballshaped tip (Fig 2).
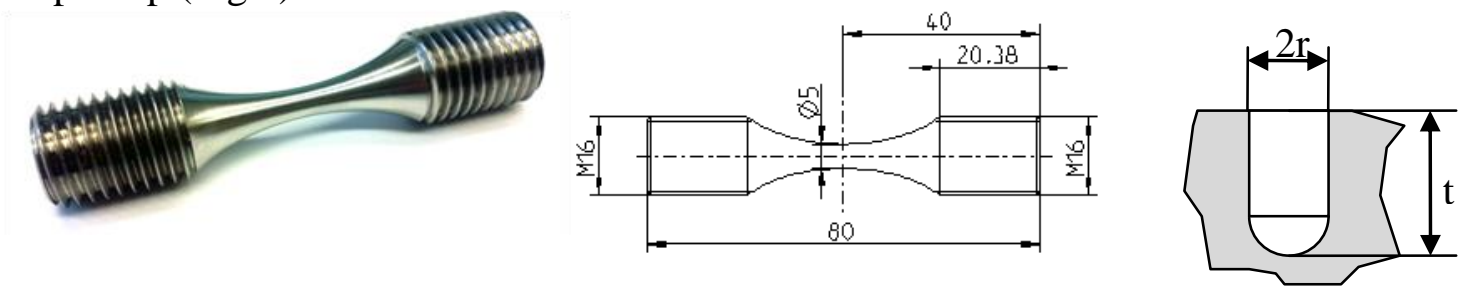

Fig. 2: Dimensions of the weak notched specimen

The load of the specimen is a uniaxial cyclic stress with a stress ratio of -1 , like it is used for the experimental work of this paper. Figure 3 shows the loaded specimen and the FEM-Model which uses symmetry. Due to this only one quarter of the specimen is modeled. The load is 
applied by a translation of the areas at the left side of the specimen about $0.01 \mathrm{~mm}$. Symmetry boundary conditions are used on the other surfaces. The specimen is meshed with hexahedra elements; the element size is variable in order to reach a very fine mesh at the corrosion pit. Finally, the mesh consists of approximately 130000 nodes and 30000 elements of second order.

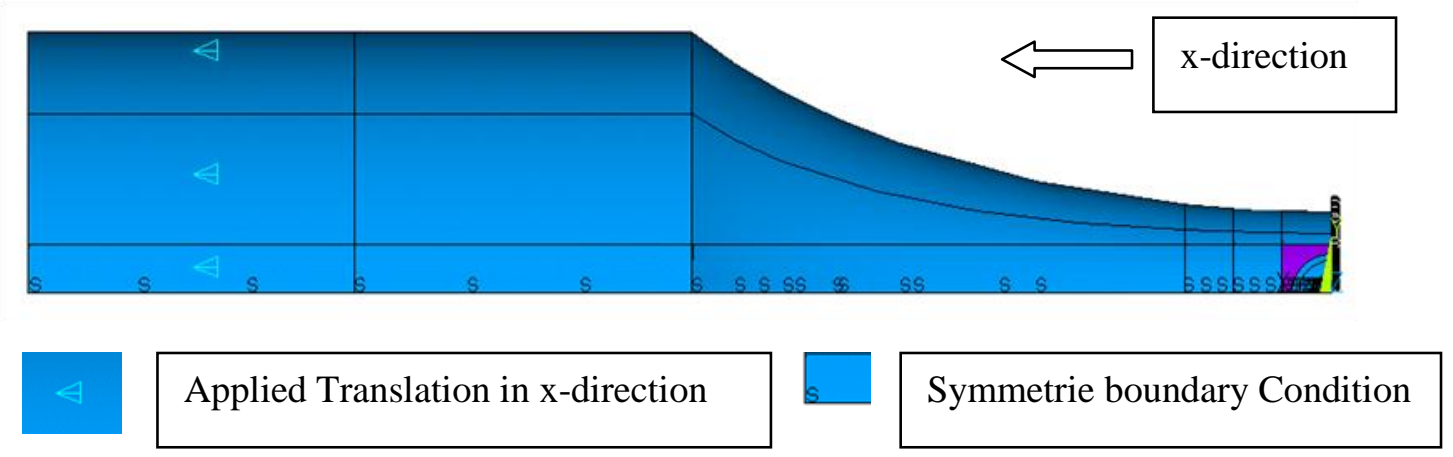

Fig. 3: FEM-Modell of the weak notched specimen

In order to retrieve the influence of different pit sizes and stress distances $a_{s}$, parameter studies have been carried out. The diameter of the corrosion pits $(20$ steps from $0.01 \mathrm{~mm}$ to $0.5 \mathrm{~mm})$ the ratio of depth and radius $(1,2,4)$ and the stress distance $(10$ steps from $0.005 \mathrm{~mm}$ to $0.3 \mathrm{~mm}$ ) were varied, at least 600 calculations have been done. Fig. 4 shows the calculated stress state of three exemplary specimens with different pit sizes. The scale of Fig. 4 is given in picture a).
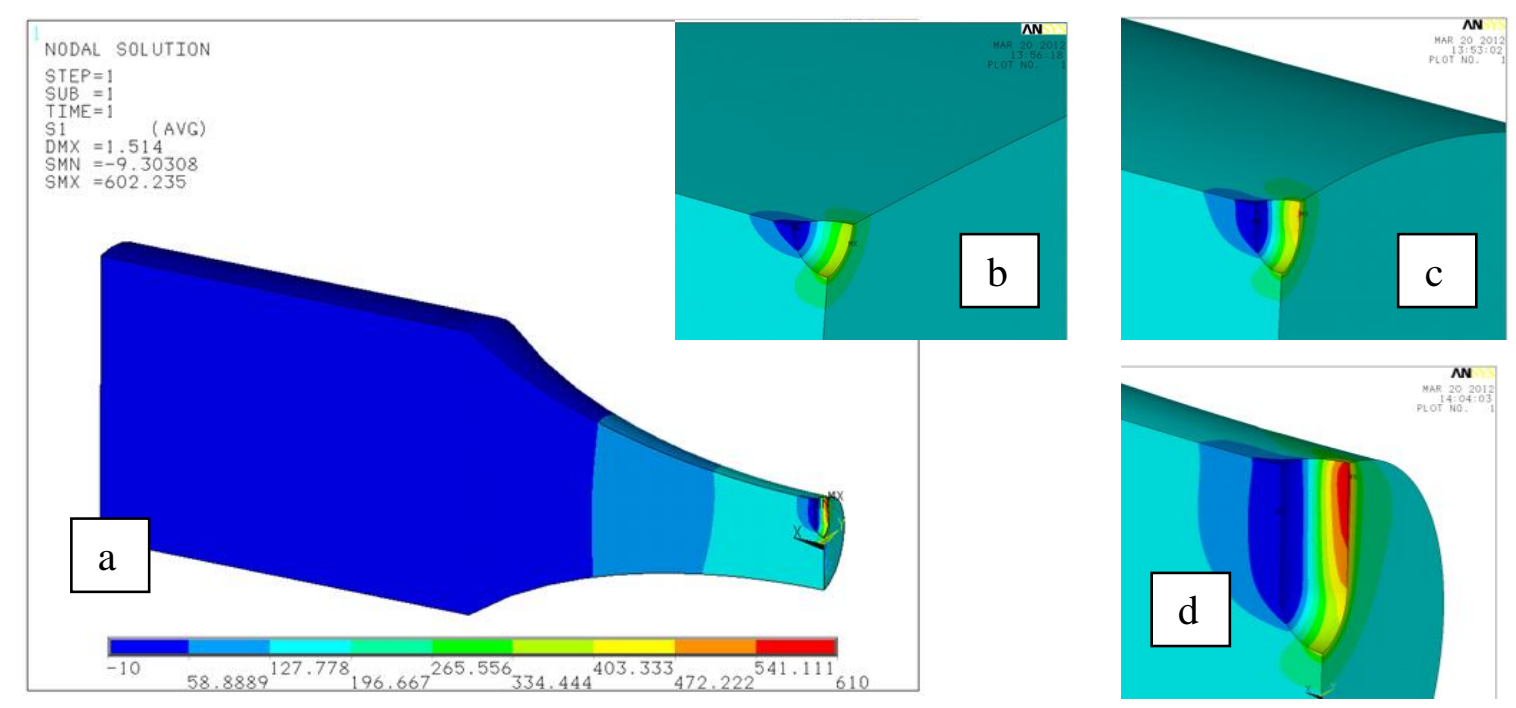

Fig. 4: First principal stress in $\mathrm{N} / \mathrm{mm}^{2}$ for three pit sizes a) and d) radius $0.5 \mathrm{~mm}$ depth $2 \mathrm{~mm} \mathrm{~b}$ ) radius $0.01 \mathrm{~mm}$ depth $0.01 \mathrm{~mm} \mathrm{c}$ ) radius $0.06 \mathrm{~mm}$ depth $0.12 \mathrm{~mm}$

According to the proposed method, the stress in distance $a_{s}$ to the surface is calculated. Generally, the nodes of the mesh are not in distance $a_{s}$ to the surface, thus a set of analysis points is generated which have the required distance. Using interpolation the stress at the analysis points is calculated.

The greatest value of the first principle stress in distance $a_{s}$ to the corrosion pit's surface is regarded as the effective stress $\sigma_{\text {eff }}$ at the corrosion pit. The nominal stress $\sigma_{\text {nom }}$ of the specimen in the middle plane is estimated by retrieving the stress at the opposite side of the specimen. The ratio of these stresses leads to the actual fatigue limit and to the notch factor of the corrosion pit $K_{C P}$. Fig 5 displays the relative actual fatigue limit $\sigma_{D a r}$, which is given by equation 2 . 
$\sigma_{D, a c t}=\sigma_{D} \cdot \frac{\sigma_{n o m}}{\sigma_{e f f}}=\frac{\sigma_{D}}{K_{C P}}$

$\sigma_{D a r}=\frac{1}{K_{C P}}=\frac{\sigma_{\text {nom }}}{\sigma_{\text {eff }}}$

The results comply with the expected behavior of the corrosion pits. There are two general tendencies: First, increasing the pit size leads to a decreasing actual fatigue limit. As mentioned above, a greater corrosion pit causes a higher peak stress and a larger area which is affected by the local stress peak. Thus a decrease of the fatigue limit can be expected. Second, a smaller stress distance leads to a decreasing actual fatigue limit. This is suitable, because a small stress distance means, that the effective stress is closer to the peak stress. The shape of the resulting curves is similar to results in literature $[8,9]$.

\section{Experimental Method}

Procedure The aim of the experimental method is to retrieve the actual fatigue limit of a component according to its corrosion state. Due to this, the corrosion state of each specimen must not change during the fatigue test, thus pre-corrosion is used. To determine the endurance strength, a modified staircase test is carried out.

The staircase test is a common method to estimate the fatigue limit of specimens and components $[1,3,9]$. It consists of a series of fatigue tests. Each fatigue test is performed with constant load amplitude and can have two different results: The specimen can break (failure) or the maximum number of loadcycles is reached without rupture of the specimen (suspension). The first fatigue test is performed with the initial load amplitude. If the specimen fails, the amplitude will be decreased, otherwise the amplitude is increased. Usually, a constant load increment $F_{\text {step }}$ is used to get an easy analysis of the test.

$F_{i+1}=F_{i}+/-F_{\text {step }}$

After the pre-corrosion of the specimens, an individual endurance strength results because of the scatter of the material properties and the size and shape of the corrosion pits. The second effect has to be considered during the staircase test and leads to the need of a modification of the test. This is the reduction of the applied load amplitude depending on the size of the corrosion pit. The reduction is calculated using the proposed calculation method for the actual fatigue limit. The parameters for the calculation can be measured (pit radius) or are estimated (stress distance $a_{s}^{*}$, depth to radius ratio $\alpha^{*}$ ). Estimated parameters are marked with an asterisk. Thus, the nominal load of each fatigue test is chosen like in a usual staircase test, but it is reduced to the reduced load according to the calculated notch factor of the corrosion pit.

$F_{i, r e d}=\frac{F_{i}}{K_{C P}\left(a_{s}^{*}, r, \alpha^{*}\right)}$

There are different options to analyze the test results. Most methods are based on the maximum-likelihood estimation (MLE). Different authors applied MLE to staircase tests and proposed simple equations for certain distribution functions [1,9]. To apply these equations, 
the load increment has to be constant. This is not given for the pre-corroded specimens. Due to this, the parameters of the distribution function are estimated by a direct MLE approach.

MLE Method The aim of the Maximum-Likelihood Method is to fit parameters of a chosen probability distribution to experimental results [10]. The so called maximum likelihood parameter $P_{M L E}$ represents the probability that the experimental results occur, depending on parameters of the chosen probability distribution. The set of parameters, which leads to the highest probability, is regarded as the best fit. In the case of staircase tests, the MaximumLikelihood approach leads to the following formula:

$P_{M L E}(\mu, \sigma)=\prod_{i} P_{\text {Fracture }, i}(\mu, \sigma) \cdot \prod_{j} P_{\text {Runout }, j}(\mu, \sigma)$

The estimation of the stress distance $a_{s}$. is also performed by the meaning of this method. In this case the MLE-parameter also depends on $a_{s}$. The maximization of $P_{M L E}$ is performed numerically.

\section{Simulated Experiments}

The proposed experimental method is tested with simulated experiments. A set of so called true parameters defines the simulated specimens, which replace the specimens of the real experiments. The experimental method is applied virtually to the simulated specimens and leads to the resulting parameters. A comparison of the true and resulting parameters allows the description of the experimental method's quality.

A number of effects have an impact on the results of a staircase test. One of these is the compliance of the parameters which are estimated before the test, and the true parameters. A poor compliance leads to a large number of fatigue tests whose load amplitude differs clearly from the Fatigue Limit, which worsens the quality of the statistical analysis. Other effects deal with the corrosion pits. Different distributions of the pit size and the test sequence (e.g. from small to big sizes or randomly) might have an impact on the results. The last group of effects is connected to the procedure of the test. These are the amount of the load increment and the type of increment. Beside the load level it is possible to change the estimated stress distance $a_{s} *$ during the test. In order to evaluate the impact of these effects, the corresponding parameters are varied within the simulated experiments.

The true and the resulting parameters consist of the stress distance and a probability distribution of the fatigue limit. To receive realistic results, the outcomes from fatigue tests [11] are taken to define the probability distribution. The stress distance was varied within their typical range [8]. The estimated parameters $a_{s}{ }^{*}$ and $\alpha^{*}$ are set to a constant value. The parameters, which correspond to the corrosion pits and the test procedure, were all varied. This leads to a number of 144 simulated versions of the experiment.

The simulation starts with the determination of the smooth specimen's fatigue limit by generating random numbers of the fatigue limit probability distribution. In order to get comparable results, all 144 simulated experiments use the same set of fatigue limits. The actual fatigue limits are calculated according to the proposed calculation method depending on the corrosion pits and the stress distance of the particular version of the experiment. The next step of the simulation is the staircase test, which is performed as an iterative sequence like the real staircase test (Fig. 6). After testing all specimens, the parameters of the resulting fatigue limit distribution and the resulting stress distance are calculated using MLE Method. The staircase test is performed 100 times using new random numbers for each run (Monte Carlo simulation) to generate a probability distribution of the estimated parameters. The comparison of this distributions leads to a well-founded statement about the suitability of the used parameters and variations of the staircase test. 


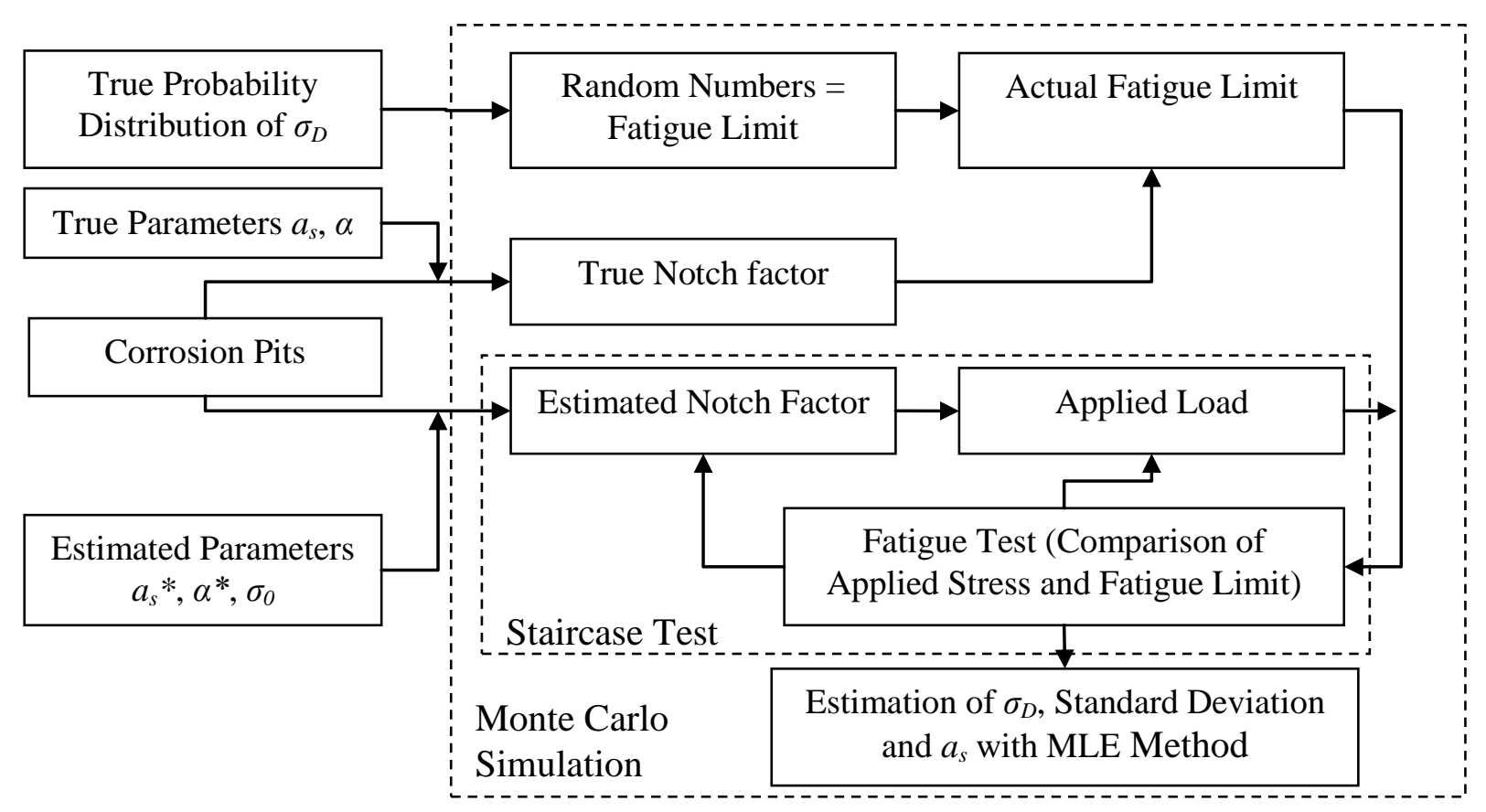

Fig. 6: Application flow of the simulated experiments

The results of the Monte Carlo simulation contain a large scatter and any of the tested types of the staircase test does not lead to reliable estimation of the fatigue limit and the stress distance Fig. 7a), Fig. 7b) and Table 1 display the great difference between the true and the calculated actual fatigue limit of one exemplary staircase test.
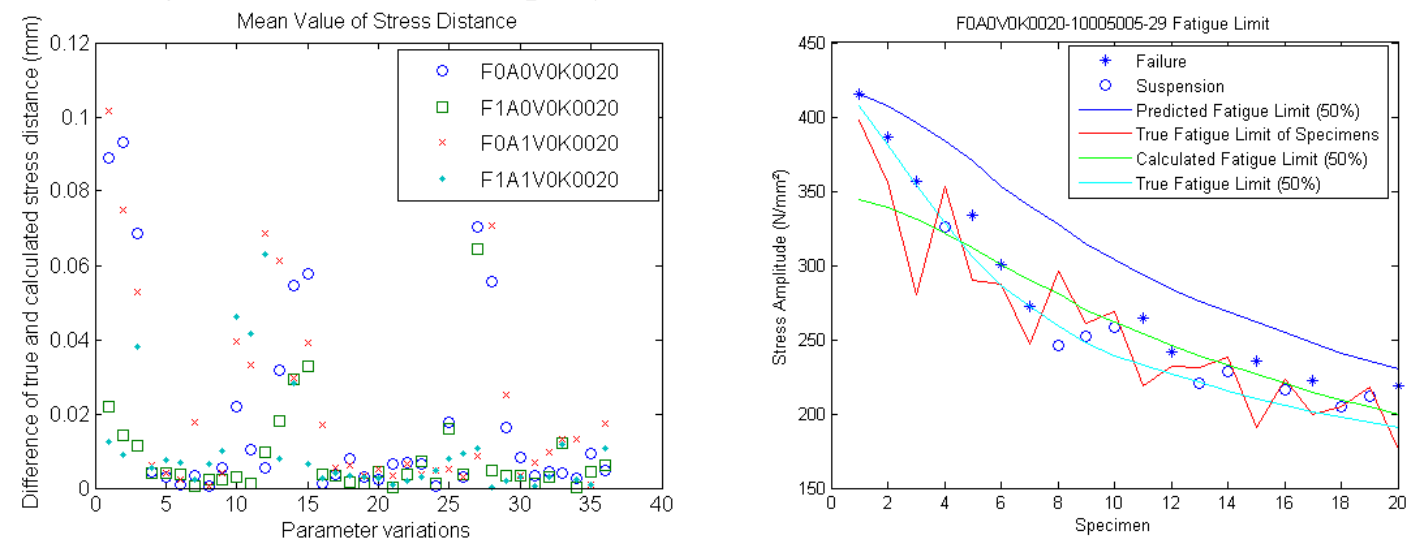

Fig. 7 (a - left), $(b-$ right): Results of simulated staircase tests

The analysis of the simulations shows that the error in the estimation of the stress distance and the fatigue depends on each other. In the case of a great error, both values are poorly estimated. Thus an additional staircase test is integrated in the application flow and all 144 experiments are performed with this modification. A new set of random numbers is used to determine the fatigue limit of the smooth specimens, the pre-corroded specimens have the same fatigue limit like in the previous simulations. Hence the test results can be compared directly.

The simulated experiments clearly show the improvement of estimating the Fatigue Limit and the stress distance. Fig. 8 shows the same graphs like Fig. 7, with the difference of including smooth specimens in the test. 

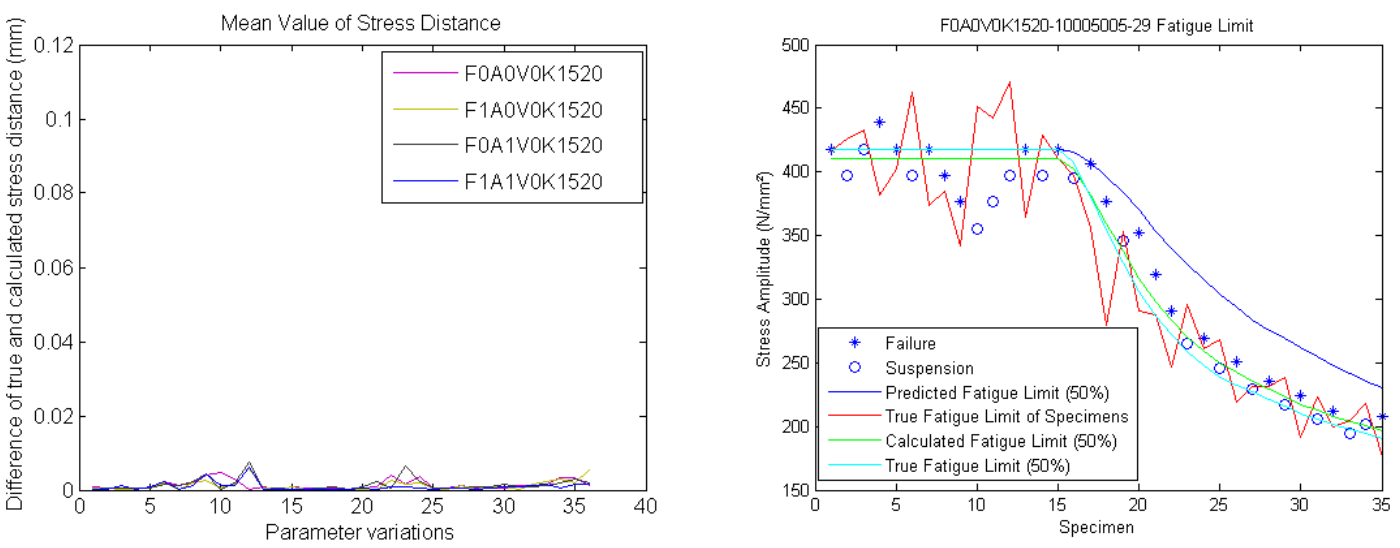

Fig. 8: Results of simulated staircase test, which include smooth specimens

Table 1: Exemplary results of simulated staircase tests

\begin{tabular}{|l|l|l|}
\hline & Fatigue Limit (50\%) & stress distance \\
\hline Only Pre-corroded specimens & $346,5 \mathrm{~N} / \mathrm{mm}^{2}$ & $0,115 \mathrm{~mm}$ \\
\hline Pre-corroded and smooth specimens & $410,5 \mathrm{~N} / \mathrm{mm}^{2}$ & $0,061 \mathrm{~mm}$ \\
\hline True Parameters & $418 \mathrm{~N} / \mathrm{mm}^{2}$ & $0,05 \mathrm{~mm}$ \\
\hline
\end{tabular}

The results of the performed simulations show, that the proposed experimental method can lead to reliably estimated parameters concerning the actual fatigue limit. Nevertheless, the simulations are based on the assumption, that the reduction of the fatigue limit can be modeled with the proposed calculation method. Due to this, the experiments have to be used to validate the calculation method as well. After all, it is suggested to test a set of smooth and pre-corroded specimens using the proposed experimental method.

\section{Experiments}

The experimental work consists of the fatigue tests with smooth specimens. As mentioned above, the results of these tests are essential for a good estimation of the stress distance and the fatigue limit. The shape and material of the specimens equals to the specimens of the presented FEM calculations. They are tested on a servo-hydraulic test-rig which applied sinusoidal loads at a frequency of $50 \mathrm{~Hz}$.

Fatigue tests without corrosion According to the results of the simulated experiments, a staircase test with smooth specimens is carried out. Hück [1] proposes a number of at last 5 to 9 specimens to retrieve the mean value of the fatigue limit, thus 10 specimens were used. In order to get a first estimation of the fatigue limit a locati test [3] was carried out. Within this test, the failure occurred at a stress amplitude of $550 \mathrm{~N} / \mathrm{mm}^{2}$. Thus, the fatigue limit was estimated to 500 $\mathrm{N} / \mathrm{mm}^{2}$. The initial load amplitude was chosen corresponding to a nominal stress amplitude of $550 \mathrm{~N} / \mathrm{mm}^{2}$, the load increment corresponding to a stress increment of $25 \mathrm{~N} / \mathrm{mm}^{2}$ (specimens 7-

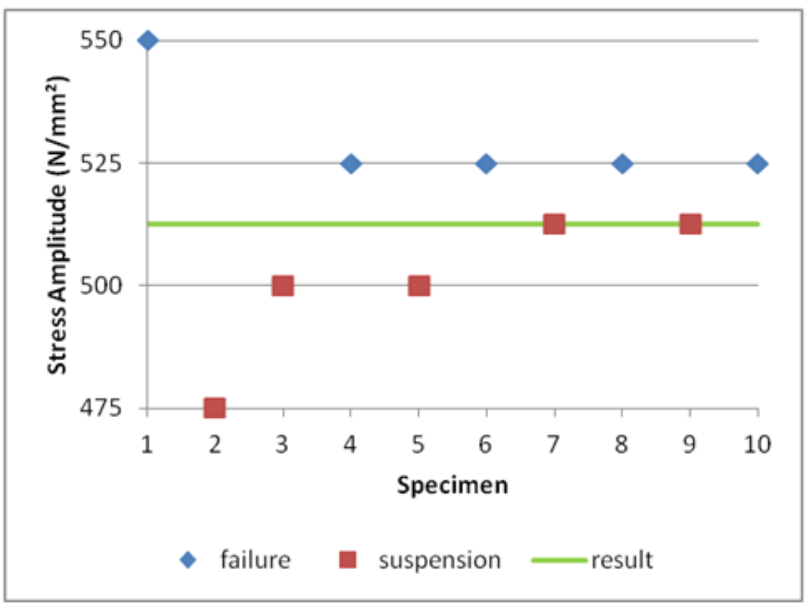

Fig. 9: Staircase test of smooth specimens 10: $12.5 \mathrm{~N} / \mathrm{mm}^{2}$ ).

The results might suggest that there is a rather sharp fatigue limit (Fig. 9). But it is more probably, that the sequence of the specimen's fatigue limit caused this test results. It is 
assumed that continuing the test would lead to failures on lower load levels due to the scatter of the specimen's fatigue limit.

According to Hück [1], a normal distribution is used for the fatigue limit. The numerical computation of equation (5) leads to a mean value of $512.6 \mathrm{~N} / \mathrm{mm}^{2}$ and a standard deviation of $0.01 \mathrm{~N} / \mathrm{mm}^{2}$. As mentioned above, the value of the standard deviation is not assumed to be close to the true standard deviation. The fatigue limit of $512.6 \mathrm{~N} / \mathrm{mm}^{2}$ is $50 \%$ greater than the calculated value using FKM guideline $\left(260 \mathrm{~N} / \mathrm{mm}^{2}\right.$ to $\left.352 \mathrm{~N} / \mathrm{mm}^{2}\right)$. This difference is comprehensible due to the survival probability of $97.5 \%$, which is used within the FKM guideline.

\section{Summary and Future Work}

In this paper a coupled experimental and numerical method for the calculation of the fatigue strength is presented. The calculation method is applied to weak notched specimens and the actual fatigue limit depending on corrosion pits is calculated. Corresponding experiments are simulated in order to evaluate the proposed approach before carrying out real experiments. Based on these simulations, the necessities of additional fatigue tests of specimens which are not pre-corroded are discovered. The corresponding experiments are been started.

The Future work will deal with the improvement of the proposed methods. The first steps are the competition of the presented fatigue tests and a review of the calculation method. Furthermore, notched specimens will be regarded. Another step is the comparison of the proposed method's results with results of fatigue tests under corrosive conditions. This will be used to include effects, which appear due to the additional interaction of corrosion and fatigue.

\section{Acknowledgements}

Parts of this work have been done within the joint research project "VMPT-Vernetzte Mehrphasentransporttechnik, Sub Sea, Off- und Onshore", funded by the German Federal Ministry for Economics and Technology, in cooperation with other German research institutes in Berlin, Hamburg and Kassel and the coordinating pump manufacturer Joh. Heinr. Bornemann GmbH.

\section{References}

[1] Hück, M.: Ein verbessertes Verfahren für die Auswertung von Treppenstufenversuchen, Werkstofftechnik 14, 406-417 (1983)

[2] Wendler-Kalsch, E.; Graefen, H.: Korrosionsschadenskunde, Springer Verlag 1998

[3] Radaj, D.: Ermüdungsfestigkeit, Springer Verlag 1995

[4] FKM Guideline for analytical strength assessment, VDMA Verlag 2003

[5] Kawai, S.; Kasai, K.: Considerations of allowable stress of corrosion fatigue, Fatigue Engn. Mater. Struct. Vol. 8, No. 2, pp. 115 - 127, 1985

[6] Hoeppner D.W.; Chandrasekaran, V.; Taylor, A.M.H.: Review of pitting corrosion fatigue models

[7] El Haddad, M.H.; Smith, K. H.; Topper, T. H.: Fatigue crack propagation of short cracks. J. Engng. Mater. Techn. (ASME) 101 (1979) 1, 42-46

[8] Fujimoto, Y.; Hamada, K.; Shintahu, E.; Pirker, G.: Inherent damage zone model for strength evaluation of small fatigue cracks. Engng. Fract. Mech. 68 (2001), 455-473

[9] Dixon, W.J.; Mood, A.M.: A method for obtaining and analyzing sensitivity data, J. Amer. Stat. Assoc. 43 (1948) 241, 109-126

[10] Kreyszig, E.: Statistische Methoden und ihre Anwendungen, Vandenhoeck und Ruprecht, (1979)

[11] Müller, J.: Schriftenreihe des Instituts für Konstruktions-technik, Ruhr Universität Bochum, Heft 86.8 